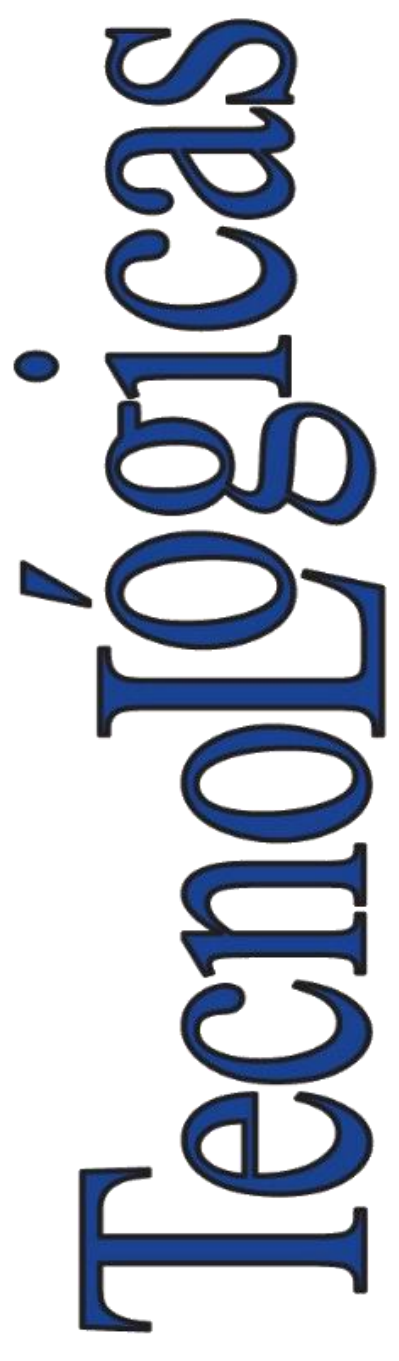

ISSN-p: 0123-7799 ISSN-e: 2256-5337

Vol. 24, nro. 51, e1924, 2021

Recibido: 13 marzo 2021 Aceptado: 11 junio 2021

Disponible: 12 julio 2021

CInstituto Tecnológico Metropolitano

Este trabajo está licenciado bajo una Licencia Internacional

Creative Commons Atribución (CC BY-NC-SA)

\section{Simulación de una antena microcinta rectangular espiral multibanda para la aplicación de captación de energía de \\ radiofrecuencia}

\section{Simulation of a Rectangular Spiral Microstrip Multiband Antenna for Radio Frequency Energy Harvest}

\author{
(iD) Edison Andrés Zapata-Ochoa ${ }^{1}$; \\ (D) Francisco López-Giraldo ${ }^{2}$; \\ iD Germán David Goéz ${ }^{3}$ \\ ${ }^{1}$ Instituto Tecnológico Metropolitano, Medellín-Colombia, \\ edisonzapata110337@correo.itm.edu.co \\ 2 Instituto Tecnológico Metropolitano, Medellín-Colombia, \\ franciscolopez@itm.edu.co \\ ${ }^{3}$ Instituto Tecnológico Metropolitano, Medellín-Colombia, \\ germangoez@itm.edu.co
}

Cómo citar / How to cite

E. A. Zapata-Ochoa; F. López-Giraldo; G. D. Goéz, "Simulación de una antena microcinta rectangular espiral multibanda para la aplicación de captación de energía de radiofrecuencia”, TecnoLógicas, vol. 24, nro. 51, e1924, 2021. https://doi.org/10.22430/22565337.1924 


\section{Resumen}

El presente estudio realizó un análisis de la influencia que ejercen las variaciones del grosor del sustrato dieléctrico y de la posición de las ranuras de microcinta creadas en el parche radiante de una antena de microcinta. El trabajo presentó la simulación mediante el software de optimización automática CST Studio, de una antena para captura de energía de radiofrecuencia empleando el substrato ARLON AD450 con constante dieléctrica de 4.5, pérdidas tangenciales de 0.035 y espesor de $3 \mathrm{~mm}$. En este diseño se aplicaron varias ranuras hasta formar una espira cuadrada. Los resultados obtenidos evidenciaron que al aumentar el espesor del sustrato que separa el plano de tierra del parche radiante, se produce una disminución en las pérdidas de retorno del parámetro $\left(S_{11}\right)$ y al mismo tiempo se da_un aumento en la ganancia de la antena. La creación de ranuras, el grosor y la ubicación de estas en el parche radiante producen un desplazamiento en la frecuencia de resonancia. Asimismo, el arreglo presentado permite frecuencias de resonancia alrededor de $1.6 \mathrm{GHz}, 2.38 \mathrm{GHz}$, $3.38 \mathrm{GHz}$ y $4.16 \mathrm{GHz}$, con una ganancia entre $2.48 \mathrm{~dB}$ y $7.66 \mathrm{~dB}$. Este diseño de antena permitió mejoras en ganancia y en el patrón de radiación. La creación de ranuras en el parche radiante modificó la distribución de corriente de superficie de la antena y generó nuevas frecuencias de resonancia. Los espacios de aire creados entre el cobre y el substrato mejoraron el rendimiento de la antena, del mismo modo que el espacio ejercido por el substrato entre el plano de tierra y el parche radiante disminuyeron las pérdidas de ganancia en la antena debido a la reducción del cobre cuando se realizan estas ranuras. La antena propuesta presenta un comportamiento multibanda inmediatamente se agregan ranuras en el parche. La evaluación de substratos y estructuras son útiles para el desarrollo de antenas de microcinta integradas para sistemas de recolección de energía de radiofrecuencia.

\section{Palabras clave}

Antenas de microcinta, ranuras de microcinta, patrón de radiación, frecuencia de resonancia.

\section{Abstract}

This study analyses the influence of variations in the thickness of the dielectric substrate and the position of the microstrip slots created in the radiating patch of a microstrip antenna. Automatic optimization software, i.e., CST Studio, was used to simulate an antenna for radio frequency energy harvesting made of ARLON AD450 substrate with a dielectric constant of 4.5, tangential losses of 0.035 and a thickness of $3 \mathrm{~mm}$. In this design, several slots were applied to form a square loop. The results show that, by increasing the thickness of the substrate that separates the ground plane from the radiating patch, the return loss of the $\left(s_{11}\right)$ parameter was reduced, and, at the same time, the antenna gain rose. Cutting out slots, as well as their thickness and location in the radiating patch, produced a shift in the antenna's resonant frequency. Likewise, the array presented here allowed resonant frequencies around $1.6 \mathrm{GHz}, 2.38 \mathrm{GHz}, 3.38 \mathrm{GHz}$, and $4.16 \mathrm{GHz}$, with a gain between $2.48 \mathrm{~dB}$ and $7.66 \mathrm{~dB}$. This antenna design produced improvements in gain and radiation pattern. Creating slots in the radiating patch modified the surface current distribution of the antenna and generated new resonant frequencies. The air gaps produced between the copper and the substrate improved the antenna's performance. Similarly, the spaces created by the substrate between the ground plane and the radiating patch decreased the gain losses in the antenna due to the reduction of the copper caused when the slots were made. The proposed antenna presented a multiband behavior immediately after the slots were added to the patch. Evaluating substrates and structures is useful for developing integrated microstrip antennas for RF energy harvesting systems.

\section{Keywords}

Microstrip antenna, microstrip slots, radiation pattern, resonance frequency. 


\section{INTRODUCCIÓN}

En años recientes, el desarrollo en tecnologías de telecomunicaciones ha aumentado, al igual que el diseño de antenas para tecnologías de 3G, 4G y 5G y el diseño de antenas para teléfonos móviles cuyo funcionamiento sea en multibandas. Actualmente, existen numerosos trabajos sobre antenas de microcinta [1], en los cuales se incluyen diferentes topologías de ranuras (slots) sobre la superficie de estas antenas [2], [3], algunos de los cuales se basan en forma de U [4] y V [5]. También, en ranuras verticales y horizontales [6], esto permite mejorar parámetros como la ganancia, la directividad y realizar cambios en el ancho de banda según sea la necesidad de aplicación. Con la ventaja de generar diseños de estructuras simples, en [7] se analizan los cambios generados en una antena de microcinta rectangular provocados por una ranura en forma de U que genera cambios de frecuencias de resonancia y mejora las propiedades de radiación sobre el parche radiante. En [8] se inserta una ranura sobre una antena rectangular monopolo y se analiza el cambio en las frecuencias de resonancia que genera una sola inserción de ranura, se varia el largo, ancho y posición de esta, se estudia el efecto que tiene esta geometría sobre los parámetros $\left(S_{11}\right)$, se dejan las bases para estudios futuros sobre este tipo de diseños y sobre cómo se puede manejar las frecuencias de resonancia de una antena con la modificación y adición de ranuras sobre el parche radiante.

$\mathrm{El}$ efecto producido por las ranuras hechas en antenas de microcinta permite tener varias bandas de operación, lo que posibilita ampliar las aplicaciones de las comunicaciones inalámbricas como Wifi, GPS, radar WiMAX, WLAN, entre otras. Uno de los usos para las antenas de microcinta es la captación de energía de radiofrecuencia (RF) del medio ambiente [9], [10]. Al igual que la transferencia de energía inalámbrica [11], [12], estas antenas son acopladas a un circuito rectificador. La combinación de estos dos elementos se conoce como rectenna, sin embargo, en este documento solo se analizará la antena como tal.

La antena es el elemento fundamental en un sistema de recolección de energía RF, ya que determina la sensibilidad del sistema para capturar las señales RF circundantes en el medio.

Para diseñar este tipo de antenas se deben tener en cuenta consideraciones como el tipo de polarización, la eficiencia, tamaño y ancho de banda de la antena. Estas consideraciones de diseño se deben al hecho de que, aunque las señales de RF son ampliamente disponibles, sus niveles de potencia en el ambiente son muy bajos [13].

Dependiendo de la aplicación y de las fuentes de energía, en el diseño de antenas para fines de recolección de energía de $\mathrm{RF}$ existen algunas de buena ganancia que a menudo tienen un diseño voluminoso usado para frecuencias más bajas, sin embargo, esto genera pérdidas de transmisión y recepción debido al acoplamiento entre la antena y el circuito rectificador [14]. Es de importancia cosechar la energía de RF de un amplio rango de bandas para hacer uso de todo el espectro que sea posible, esto se puede lograr usando antenas de banda ancha [15]. Aunque dichas antenas son de baja ganancia, también se generan complicaciones al adaptar un circuito rectificador [16]. Además, existen otras pérdidas en la inserción de elementos en el diseño del circuito rectificador. Otros autores prefieren las antenas de banda estrecha para la recolección de energía de RF debido a que la ganancia de estas antenas es alta en una sola dirección, esto hace posible recolectar la mayor cantidad de energía del ambiente si se conoce la ubicación de la fuente emisora [17], [18], [19].

En vista de que la geometría es relativamente simple, las antenas en espiral poseen características atractivas como fácil fabricación, bajo costo, ser livianas y de fácil integración con circuitos de microondas y sensores de bajo consumo de potencia. Igualmente, pueden ser utilizadas en aplicaciones móviles debido a que sus características la hacen antena multibanda. Se han construido algunas antenas de microcinta con diseño en forma de espiral circular o cuadrada, en su mayoría para ser utilizadas en la transferencia de energía por 
acoplamiento inductivo [20], las cuales se utilizan para carga de implantes médicos [21] y carga de baterías a corta distancia [22], así como para integrar la conexión entre tarjetas para identificación por radiofrecuencia (RFID, por sus siglas en inglés) y futuros chips de banda ultra ancha (UWB, por sus siglas en inglés) y frecuencia ultra alta (UHF, por sus siglas en inglés) [23].

Debido a las características de polarización circular y a las características de antena multibanda para aplicaciones de captación de energía de $\mathrm{RF}$, este documento tiene como objetivo investigar el diseño de antenas de microcinta con ranuras en espira rectangular en el parche resonante para lograr una buena ganancia y así recolectar la mayor cantidad de energía circundante en el ambiente. El diseño final de la antena se presenta en la Figura 1: a) parte frontal (parche radiante de la antena, diseño en espiral cuadrada), y b) parte posterior (plano de tierra de la antena). De igual forma, las dimensiones de la antena propuesta se presentan en la Tabla 1.

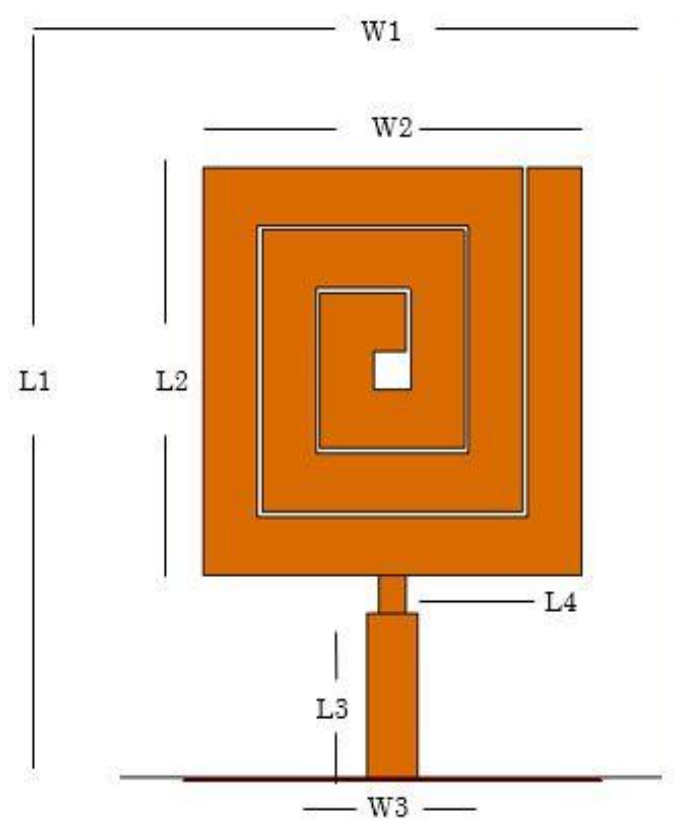

a)

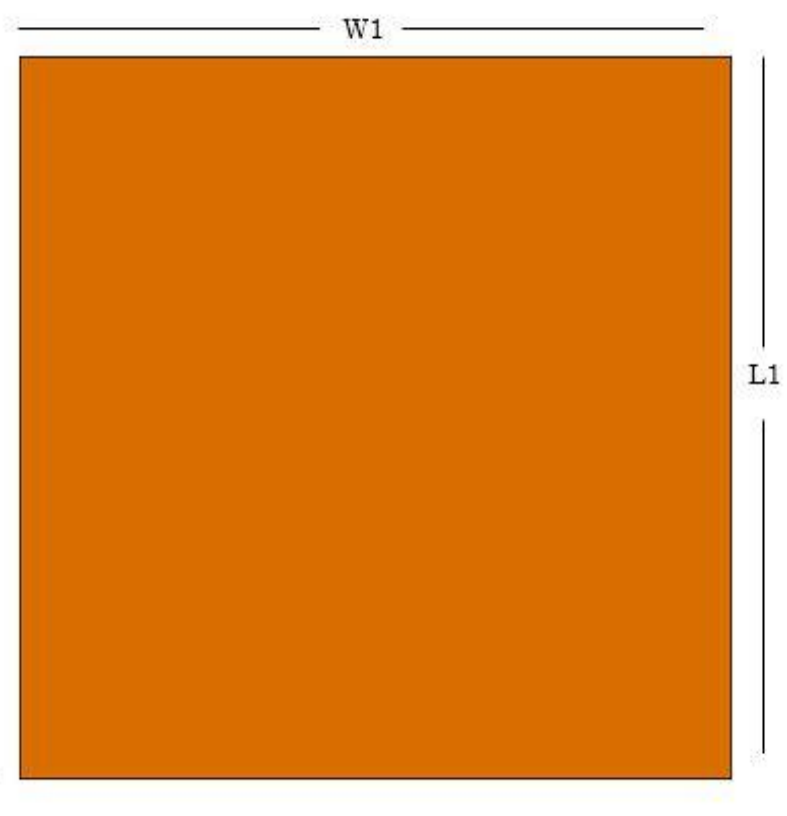

b)

Figura 1. Diseño de la antena propuesta, a) parche radiante y b) plano de tierra. Fuente: elaboración propia.

Tabla 1. Dimensiones de la antena. Fuente: elaboración propia.

\begin{tabular}{lc}
\hline \multicolumn{1}{c}{ Parámetro } & Dimensión (mm) \\
\hline Ancho del substrato (W1) & 90.0 \\
Largo del substrato (L1) & 81.4 \\
Ancho del parche (W2) & 42.0 \\
Largo del parche (L2) & 42.0 \\
Largo línea de alimentación (L3) & 16.7 \\
Ancho línea de alimentación (F3) & 5.67 \\
Largo del stub (L4) & 4.50 \\
Ancho del stub (w3) & 3.00 \\
Espesor del substrato (h) & 3.00 \\
\hline
\end{tabular}




\section{DISEÑO DE LA ESTRUCTURA}

El diseño y la simulación de esta antena se realiza con el software CST Studio suite. Se presenta inicialmente una estructura rectangular convencional, alimentada por una línea de microcinta acoplada a $50 \Omega$, que a su vez sería acoplada a un conector SMA Jack hembra.

Las características principales del substrato ARLON AD450 son:

Constante dieléctrica $=4.5$

Pérdidas tangenciales $=0.0035$

Espesor de dieléctrico $=3 \mathrm{~mm}$, como característica a destacar.

Altura del cobre $=0.035 \mathrm{~mm}$

En la Figura 2 se presenta el parche radiante con las dimensiones de las ranuras que forman la espiral cuadrada, estas dimensiones se especifican en la Tabla 2.

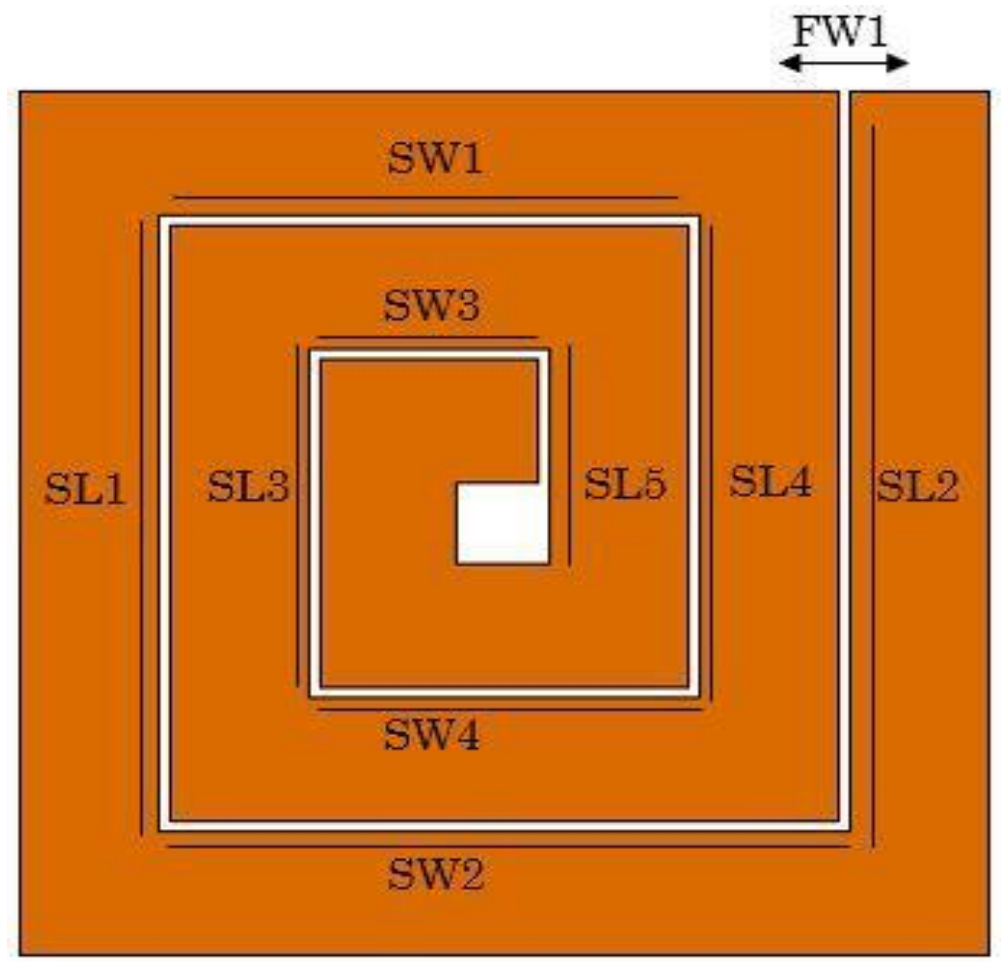

Figura 2. Dimensiones de ranura espiral en parche radiante. Fuente: elaboración propia.

Tabla 2. Dimensiones de ranuras. Fuente: elaboración propia.

\begin{tabular}{cc}
\hline Parámetro & Dimensión $(\mathrm{mm})$ \\
\hline (SW1) & 23.5 \\
(SW2) & 30.0 \\
(SW3) & 10.5 \\
(SW4) & 17.0 \\
(SL1) & 30.0 \\
(SL2) & 36.0 \\
(SL3) & 17.0 \\
(SL4) & 23.5 \\
(SL5) & 10.5 \\
(FW) & 0.50
\end{tabular}


El substrato utilizado para modelar esta antena es ARLON AD450, el cual es utilizado como base para dispositivos electrónicos y circuitos integrados de alta frecuencia, y por el grosor del dieléctrico y la separación que ejerce entre el plano de tierra y el parche radiante.

Este substrato podría sustituir al FR4. Substratos más gruesos disminuyen las pérdidas de ganancia debido a la reducción del cobre por el diseño de la antena [24] y la línea de transmisión se diseña para una impedancia de $50 \Omega$. Las ecuaciones (1) a (4) determinan las dimensiones del parche rectangular.

$$
\begin{gathered}
w=\frac{c}{2 f \sqrt{\frac{\varepsilon_{r}+1}{2}}} \\
L=\frac{1}{2 f_{r} \sqrt{\varepsilon_{e f f}} \sqrt{\mu_{0} \varepsilon_{0}}}-2 \Delta L \\
\varepsilon_{e f f}=\frac{1}{2}\left(\varepsilon_{r}+1\right)+\frac{1}{2}\left(\varepsilon_{r}-1\right)\left[\frac{1}{\sqrt{1+12\left(\frac{h}{w}\right)}}\right] \\
\Delta L=0.412 h\left[\left(\frac{\varepsilon_{e f f}+0.3}{\varepsilon_{e f f}-0.258}\right)\left(\frac{\left(\frac{w}{h}+0.264\right)}{\left(\frac{w}{h}+0.813\right)}\right)\right]
\end{gathered}
$$

Utilizando (1) y (2) se calcula el ancho y largo del parche. De las anteriores ecuaciones se calcula que la $\varepsilon_{e f f}, \mu_{0}$ y $\Delta L$ es la constante dieléctrica efectiva, la permeabilidad del espacio libre y la longitud de la extensión, respectivamente, $c$ es la velocidad $\left(3 \times 10^{8} \frac{\mathrm{m}}{\mathrm{s}}\right)$, $f$ es la frecuencia de operación y $\varepsilon_{r}$ es el índice efectivo del substrato. La optimización en el gráfico de parámetros de pérdida de retorno se realiza modificando los valores de dimensión utilizando la función de barrido paramétrico en Computer Simulation Technology Microwave Studio (CST-MWS).

\subsection{Metodología}

Los cálculos iniciales para la antena propuesta se realizan para un diseño destinado al rango de $2.4 \mathrm{GHz}$. A partir de los resultados preliminares, se hace una ranura a lo largo del parche radiante con dimensiones de $10 \mathrm{~mm}$ x $1 \mathrm{~mm}$ para ajustar la frecuencia deseada. Sin embargo, al no lograr que las bandas de resonancia iniciales se desplazaran hacia la izquierda buscando la frecuencia deseada, se replica la ranura inicial formando una figura en forma de rectángulo dentro del parche radiante; al no obtener la frecuencia deseada, se opta por formar una espira cuadrada. El software CST permite construir diferentes figuras que se pueden encajar en el diseño planteado, se forma la espiral cuadrada con iguales características de grosor que el cobre empleado en el parche radiante se procede a incrustar esta espira en el parche y se utiliza la herramienta sustraer para perforar dicho parche en el cual se genera la ranura en espira. En la Figura 3 se aprecian las dimensiones iniciales de esta. En la simulación se aplicó el método de dominio de elementos finitos (FEM, por sus siglas en inglés) 
y un solucionador de dominio de frecuencia donde se calculan estructuras resonantes como filtros, sistemas multipuertos conectores y arreglos.

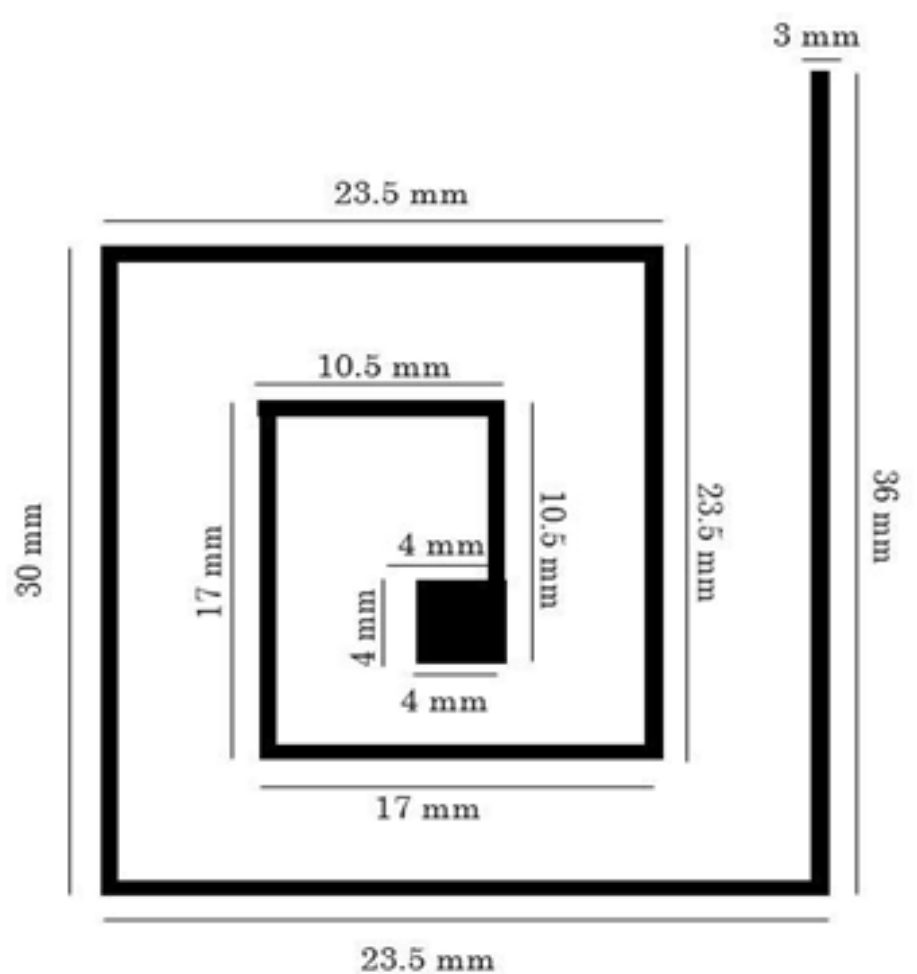

Figura 3. Dimensiones de ranura espiral en parche radiante. Fuente: elaboración propia.

Según [25], al realizar ranuras en el plano de tierra se produce una perturbación de los campos electromagnéticos alrededor de estas, los campos eléctricos atrapados generan un efecto capacitivo, mientras que las corrientes superficiales en torno de la ranura ocasionan un efecto inductivo que, a su vez, genera características resonantes, produciendo efectos de filtro. Esto también aplica al realizar ranuras en el parche radiante. Al variar el ancho de las ranuras, la capacitancia y la inductancia cambian, lo que genera como resultado un desplazamiento de la frecuencia de resonancia hacia la izquierda o hacia la derecha, según sea la necesidad [26]. En óptica se establece que, cuando se perturba un frente de onda en cualquier punto esta genera una onda secundaria. Esta perturbación se puede encontrar en un punto posterior sumando las contribuciones de las ondas individuales. Este mismo principio se aplica a las ondas electromagnéticas emitidas, en este caso, por las antenas de microcinta, lo que se conoce como el principio de Babinet [27], [28]. El procedimiento de variar las dimensiones de las ranuras solo se repite alterando el ancho de la ranura hasta lograr la frecuencia deseada, donde, a la vez, se obtuvo una antena multibanda.

\subsection{Resultados de la caracterización}

A continuación, se presenta el diseño con sus parámetros iniciales y se analizan algunos resultados obtenidos que permiten observar la variación que experimentan algunos de los parámetros más significativos de la antena a medida que se realizan ranuras en el parche radiante. Generalmente, cuando se diseñan antenas de microcinta se coloca una pared reflectante en la parte posterior de las mismas para suprimir la radiación hacia atrás cuando se requiere un patrón de radiación en una sola dirección. Esta pared se conoce como plano de 
tierra, es una lámina usualmente de cobre conectada al punto de tierra del circuito, se utilizan grandes planos de tierra para reducir el ruido eléctrico y evitar la diafonía.

El parche radiante es una antena de bajo perfil ubicada encima de una superficie. Consiste en un plano de metal formado por cualquier figura geométrica, el plano de tierra y el parche radiante forman una estructura resonante de línea de transmisión. Los parámetros $(S)$ describen la relación entrada-salida entre puertos o terminales en un sistema eléctrico. Un puerto se define como cualquier lugar donde podamos suministrar voltaje y corriente, el parámetro $\left(S_{11}\right)$ proporciona información de la transferencia de potencia del generador hacia la antena, también se conoce como la potencia reflejada en el puerto 1, cuando se genera una onda incidente en el puerto.

Como se puede apreciar en la Figura 4, la antena sin ranuras presenta tres bandas de frecuencia en $5.8 \mathrm{GHz}, 6.5 \mathrm{GHz}$ y $7.9 \mathrm{GHz}$ y los parámetros $\left(S_{11}\right)$ indicando la medida de acoplamiento de la impedancia de la antena.

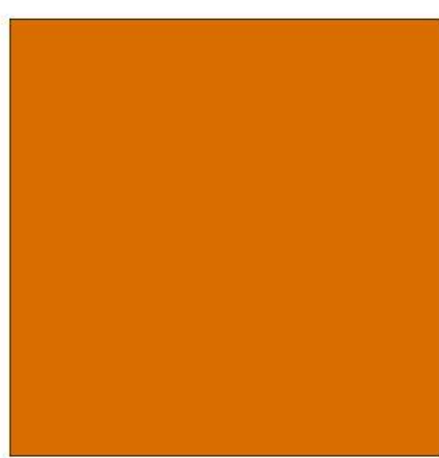

a)

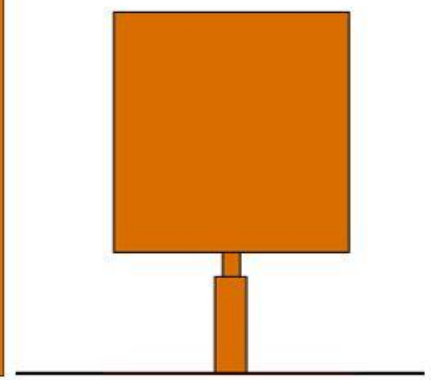

b)

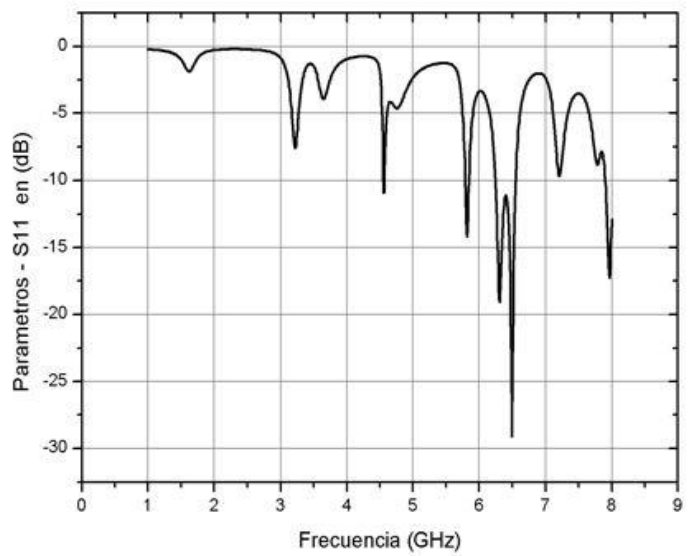

c)

Figura 4. Diseño inicial de la antena propuesta, a) plano de tierra, b) parche radiante inicial y c) parámetros $\left(S_{11}\right)$. Fuente: elaboración propia.

El patrón de radiación es un gráfico que representa la fuerza de los campos electromagnéticos emitidos por una antena. La ganancia es la característica más importante de una antena. Es la potencia de amplificación de la señal, representa la relación entre la intensidad de campo que produce una antena en un punto determinado. La unidad para medir la ganancia es el decibelio (dB). La frecuencia de resonancia de una antena es aquella frecuencia en la que se entrega la máxima potencia al parche radiante, es decir, cuando ocurre la mejor adaptación de impedancia entre la línea de alimentación y el parche. En la Figura 5 se presentan los patrones de radiación simulados de cada frecuencia con su respectiva ganancia en $\mathrm{dB}$, indicada en la barra de colores ubicada a la derecha de cada figura, siendo la ganancia más baja representada en colores fríos y la ganancia más alta interpretada por colores más cálidos.

El tipo de ranura seleccionada es de forma lineal a lo largo de la estructura del parche radiante, estas se duplican hasta formar una espira, permitiendo una distribución de corriente de superficie sobre el parche radiante Figura 5. 


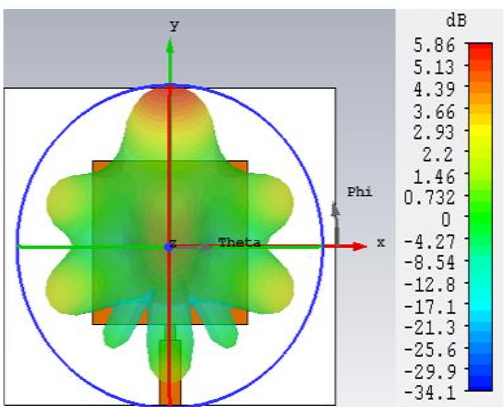

a)

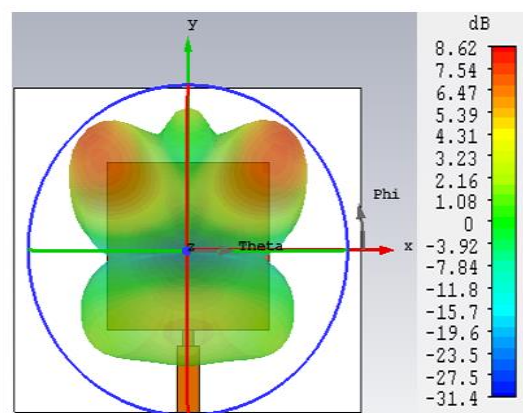

b)

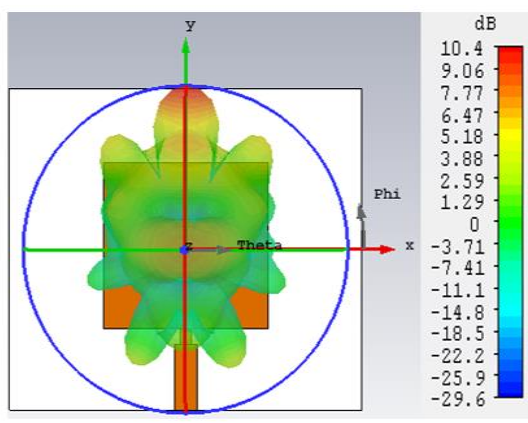

c)

Figura 5. Patrón de radiación en $3 \mathrm{D}$ e indicadores de ganancia en dB de cada frecuencia de operación diseño inicial, a) $5.8 \mathrm{GHz}$, b) $6.5 \mathrm{GHz}$ y c) $7.9 \mathrm{GHz}$. Fuente: elaboración propia.

En la Figura 6a) se observa el flujo de corriente de superficie que circula el borde del parche radiante cuando este no presenta ninguna perturbación por ranura; en la Figura 6b), el flujo de corriente aumenta y comienza a llenar el vacío producido por la ranura en el parche radiante; en la Figura 6c), el flujo de corriente va llenando los vacíos producidos por la nueva ranura.

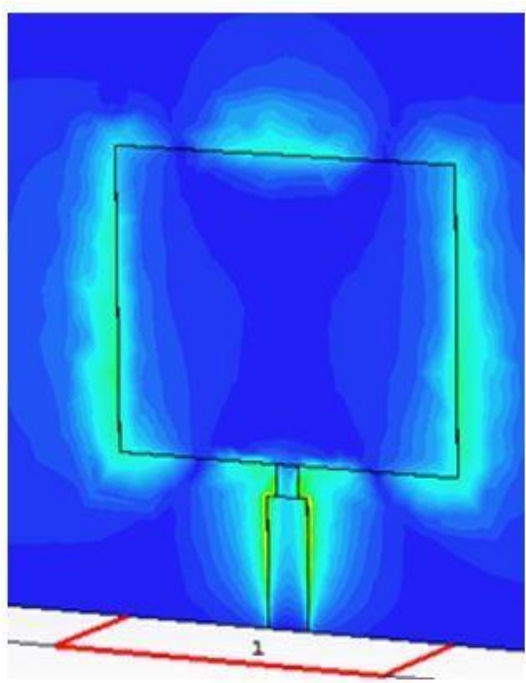

a)

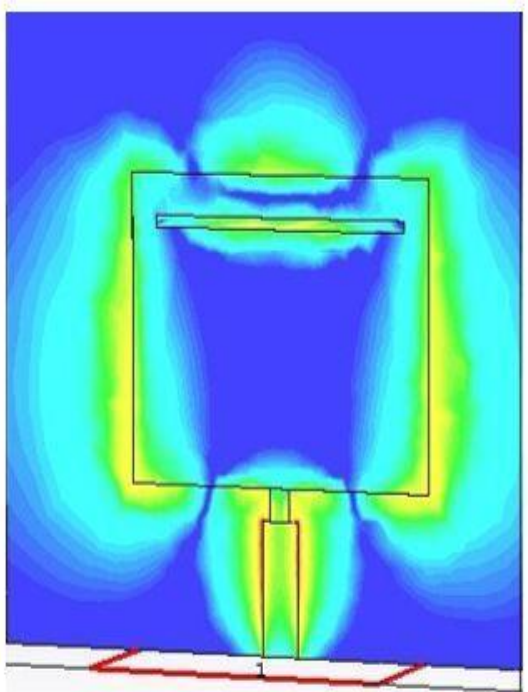

b)

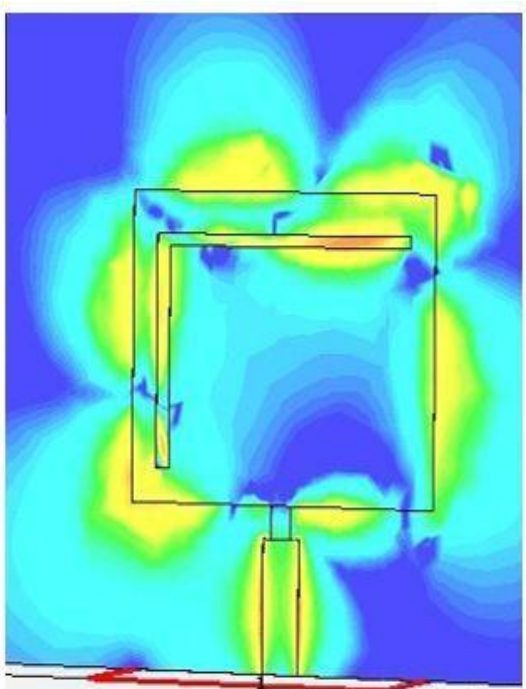

c)

Figura 6. Flujo de corriente de superficie en el parche radiante: a) parche sin ranura, b) parce radiante con ranura horizontal y c) parche radiante con ranura horizontal y vertical izquierda. Fuente: elaboración propia.

\subsection{Resultados obtenidos debido a la influencia del grosor del substrato y de las ranuras en el parche radiante}

A continuación, se pueden observar los cambios que presenta la simulación debido al efecto producido por el diseño de la espira rectangular en el coeficiente de reflexión $\left(S_{11}\right)$, generando cierta tendencia al surgimiento de nuevas frecuencias de resonancia. El plano de tierra se mantiene constante sin ninguna perturbación. Las ranuras modifican la distribución de corriente de la antena y aumentan la longitud eléctrica de esta. Aunque los cambios en la posición de las ranuras y el grosor de esta pudieron alterar las impedancias de entrada en las diferentes frecuencias de trabajo, estas permanecieron casi constantes cuando las ranuras son de $1 \mathrm{~mm}$ de grosor. La perturbación en las distribuciones de corriente superficial aumentan con la longitud de las ranuras [29]. En la Figura 7 se presentan los parámetros $\left(S_{11}\right)$ obtenidos 
cuando se realiza la variación en el ancho de la ranura de la espira, el cual es de $3 \mathrm{~mm}$. Este tipo de ranura ocasiona el desplazamiento de las bandas de frecuencia hacia la izquierda con la banda en $3.9 \mathrm{GHz}$ con -25 pérdidas por dB y la presencia de otras bandas de interés en $2.5 \mathrm{GHz}$ y $1.9 \mathrm{GHz}$.

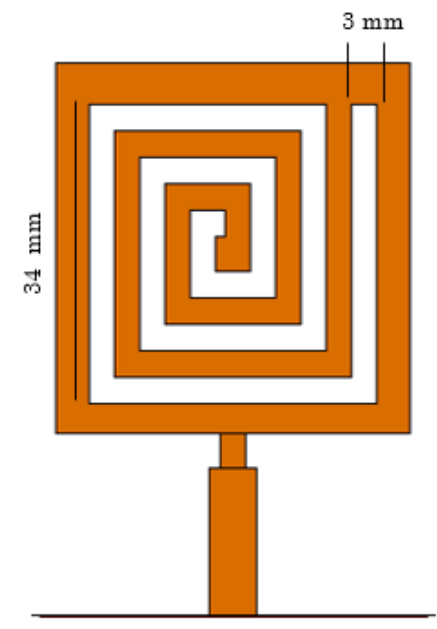

a)

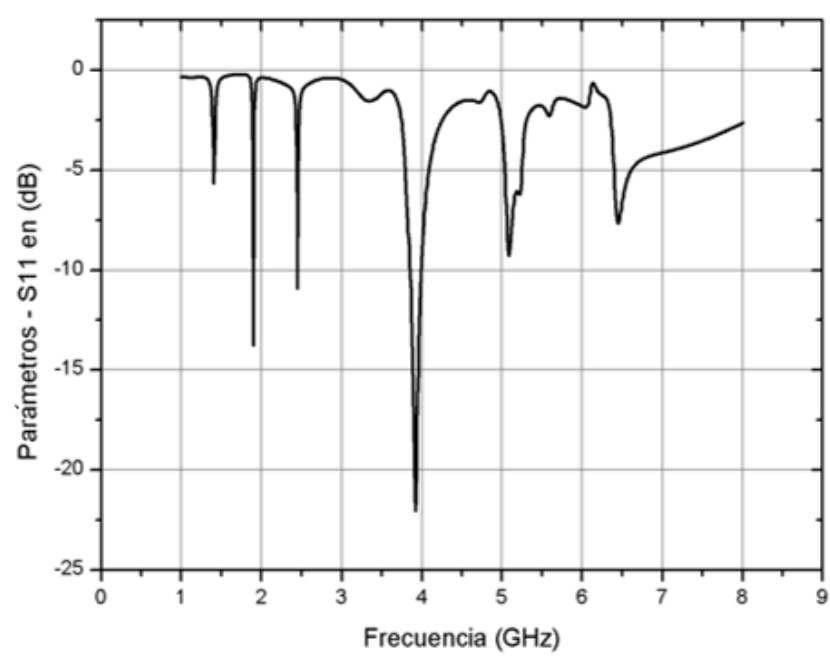

b)

Figura 7. a) Diseño de antena con ranura de $3 \mathrm{~mm}$ en el parche radiante, y b) parámetros $\left(S_{11}\right)$ simulados Fuente: elaboración propia.

La Figura 8 presenta respuesta de múltiples bandas de frecuencia, aumentando las pérdidas por $\mathrm{dB}$ en la frecuencia antes mencionada de $3.9 \mathrm{GHz}$ que continúa desplazándose hacia la izquierda; sin embargo, se aprecia el surgimiento de nuevas bandas de operación hacia la izquierda en $1 \mathrm{GHz}$ y hacia la derecha de $6 \mathrm{GHz}$, la más notoria, apreciándose que las bandas de operación son más directivas y de menos ancho de banda.

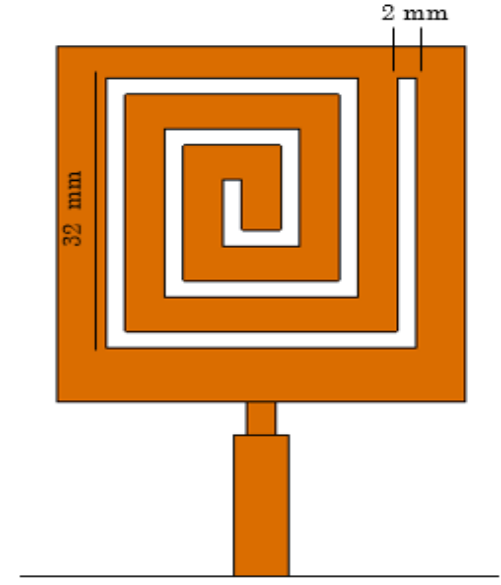

a)

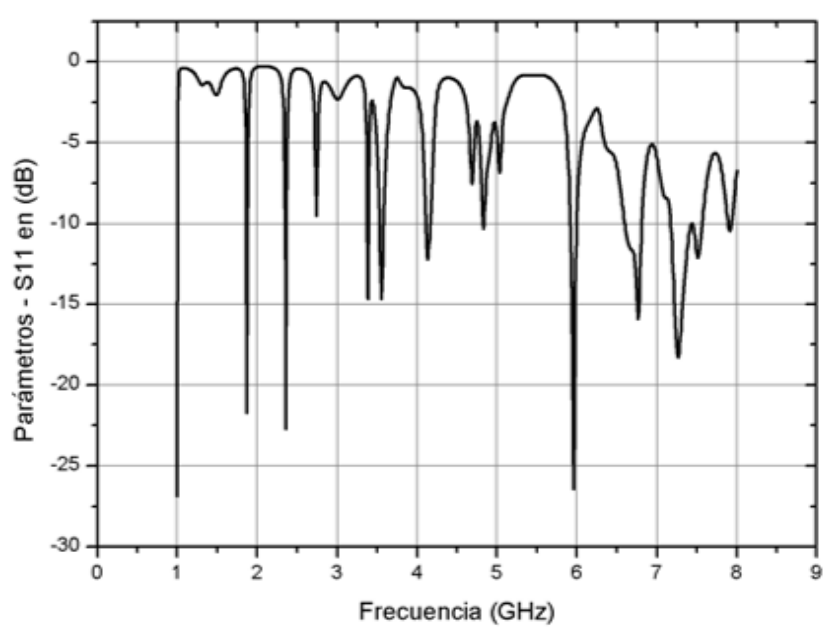

b)

Figura 8. a) Diseño de antena con ranura de $2 \mathrm{~mm} \mathrm{y} \mathrm{b)} \mathrm{parámetros}\left(S_{11}\right)$ simulados Fuente: elaboración propia.

Al igual que los parámetros $\left(S_{11}\right)$ de la Figura 8, la Figura 9 presenta los parámetros $\left(S_{11}\right)$ de la antena multibanda con frecuencias de operación de importancia en bandas comunes que 
van desde $1.86 \mathrm{GHz}, 2.38 \mathrm{GHz}, 3.38 \mathrm{GHz}$ y $4.16 \mathrm{GHz}$. No se realiza la presentación del diseño de antena con ranura de $1 \mathrm{~mm}$ debido a que presentó valores similares al diseño de la antena con ranura de $2 \mathrm{~mm}$.
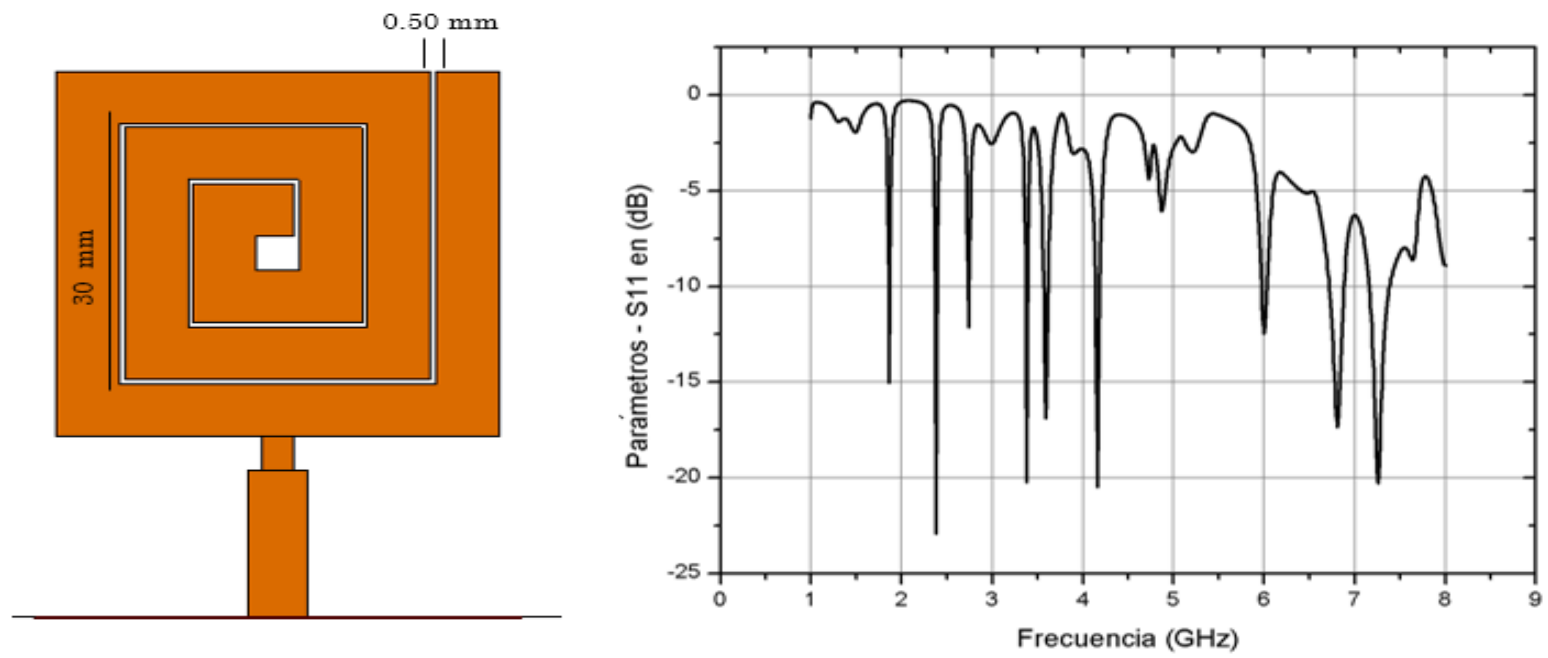

a)

b)

Figura 9. a) Diseño de antena con ranura de $0.5 \mathrm{~mm}$ en el parche radiante y b) parámetros $\left(S_{11}\right)$ simulados Fuente: elaboración propia.

La Figura 10 presenta el patrón de radiación en 3D junto a la ganancia en dB de la simulación presentada en la Figura 9 de cada frecuencia de operación de interés. Los patrones de radiación no representan cambios significativos y permanecen iguales en las diferentes frecuencias evaluadas. Como resultado de la generación de varias ranuras formando una espira cuadrada, se observa que las frecuencias de operación poseen buena ganancia.

En la Figura 11 se observa que el comportamiento de la distribución de corriente superficial vectorial en el parche radiante gira sincrónicamente debido a las ranuras en forma de espira, llenando los espacios y generando una polarización circular hacia la derecha y hacia la izquierda. En la Figura 11a), la corriente superficial comienza a tomar fuerza a partir de la ranura interna. En la Figura 11b), las corrientes superficiales se concentran a lo largo de las ranuras del parche y la dirección opuesta conduce a una banda de operación WLAN al igual que la Figura 11c) y la Figura 11d). Los colores cálidos indican mayor concentración de corriente, los colores fríos indican menor circulación de corriente por las ranuras. 

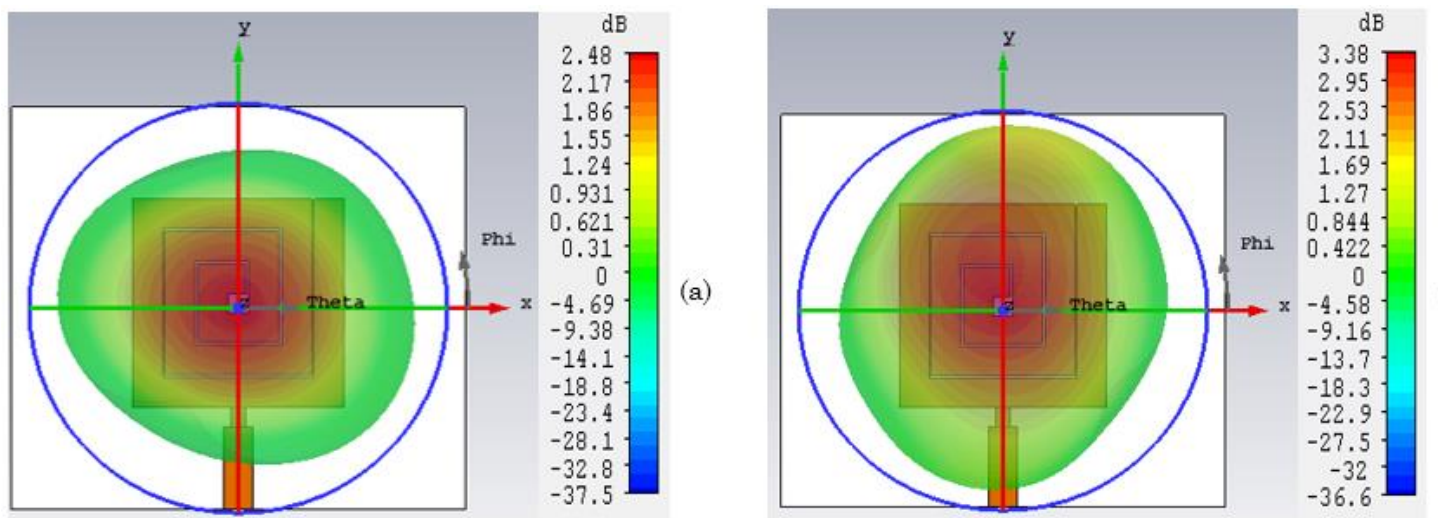

(b)
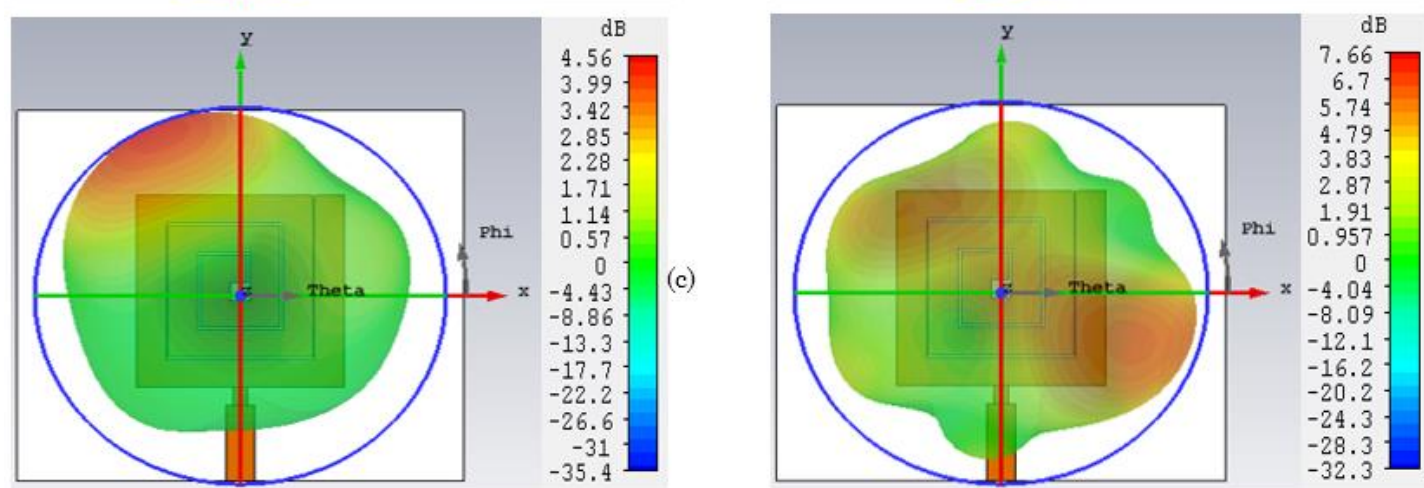

(d)

Figura 10. Patrón de radiación en 3D para las diferentes frecuencias de operación evaluadas con ranura de $0.5 \mathrm{~mm}$ y ganancia en $\mathrm{dB}$ indicada en la barra de calor, a) $2.48 \mathrm{GHz}$, b) $3.38 \mathrm{GHz}, \mathrm{c}) 4.56 \mathrm{GHz}$ y d) $7.66 \mathrm{GHz}$ Fuente: elaboración propia.

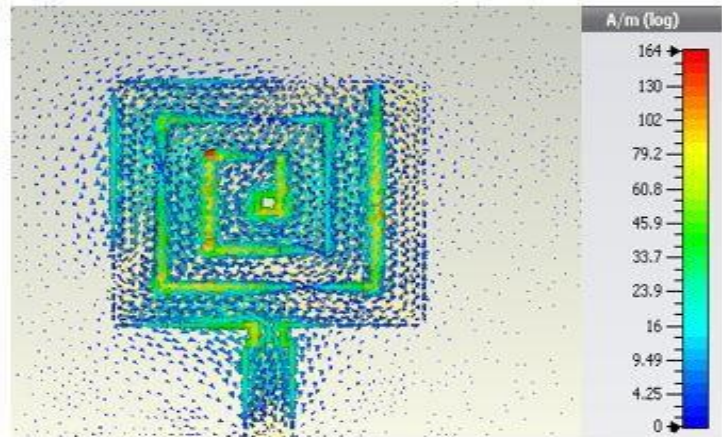

(a) a $1.8 \mathrm{GHz}$

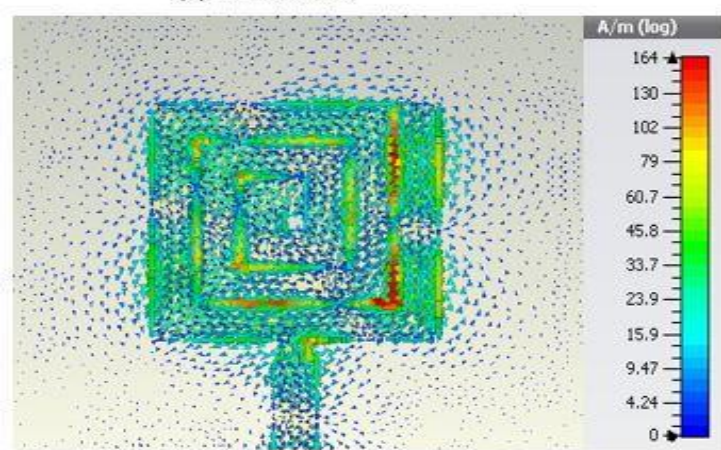

(c) a $3.38 \mathrm{GHz}$

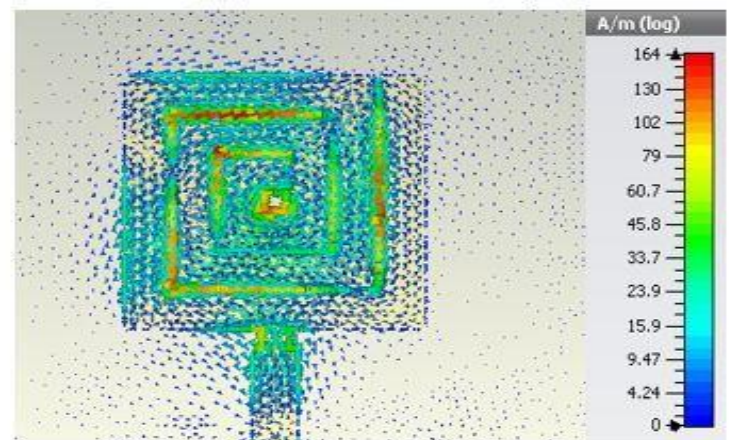

(b) a $2.38 \mathrm{GHz}$

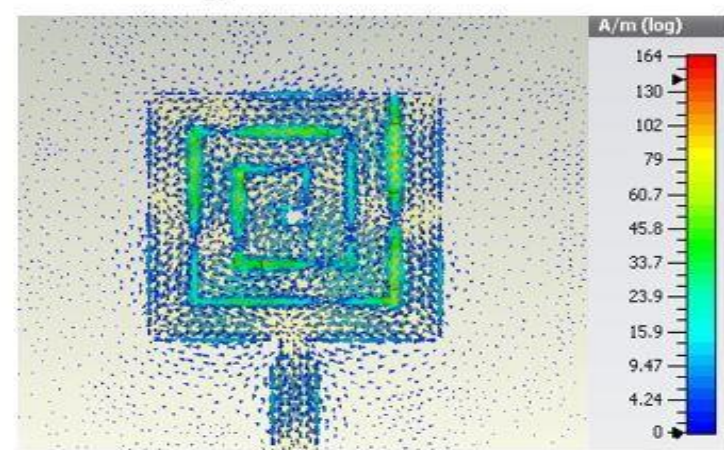

(d) a $4.16 \mathrm{GHz}$

Figura 11. Distribuciones de corriente de la antena propuesta para las frecuencias de operación evaluadas Fuente: elaboración propia. 


\section{PARÁMETROS DE ESTUDIO}

Las frecuencias de ondas milimétricas poseen características de propagación que necesitan desarrollar sistemas de comunicación de alta eficiencia; el rendimiento de estos depende en su mayoría de la estructura de la antena y se deben afrontar retos para el mejoramiento de la ganancia y el acoplamiento con otros sistemas [30]. A causa de las altas pérdidas que sufren las frecuencias durante su trayectoria, se deben usar antenas de alta ganancia al igual que antenas directivas para reducir las cargas de trayectos múltiples al limitar los ángulos desde los cuales se emite y captura la radiación.

Cuando se desconoce la posición de los terminales se utilizan antenas de haz orientables, estas son las indicadas para este trabajo, ya que la polarización de las antenas tiene gran influencia en los enlaces. Cuando se desconoce la ubicación de los terminales, las antenas con diferentes polarizaciones son las que permiten el funcionamiento de este, mejorando el sistema terminal. De este modo, las antenas con diferentes polarizaciones son las que permiten el funcionamiento del enlace, mejorando el sistema.

\subsection{Polarización}

La polarización es el plano en el que vibran las ondas electromagnéticas; por lo general, las antenas solo reciben o transmiten con una polarización particular, entre estas están:

La polarización lineal, que es la forma más común de polarización de las antenas. Se caracteriza por el hecho de que toda la radiación está en un plano, de ahí el término lineal; y la polarización circular, la cual se obtiene induciendo dos modos de corrientes ortogonales de igual amplitud y cuadratura en fase. Esto se logra generando ranuras en el parche radiante. Este método perturba los modos creando una fase de $90^{\circ}$ en la frecuencia de operación.

Dependiendo de la estructura y de la forma de las ranuras, la polarización circular resultante será derecha o izquierda, como se pudo ver en la Figura 11.

\subsection{Diagramas de radiación}

El diagrama de radiación de una antena se define como la representación gráfica de las características de radiación en función de la dirección angular. Habitualmente se utiliza un sistema de coordenadas esféricas $(\gamma, \theta, \varnothing)$, como se observa en la Figura 12a), donde también se puede ver de forma animada. Asimismo, es factible representar el campo eléctrico, magnético o la densidad de potencia radiada. Dado que los campos son magnitudes vectoriales se puede representar el módulo o la fase de sus componentes. Igualmente, las formas de representación tal vez sean tridimensionales o bidimensionales, en escalas lineal o logarítmica. La Figura 12b) es la representación tridimensional de los campos radiados por una antena. 


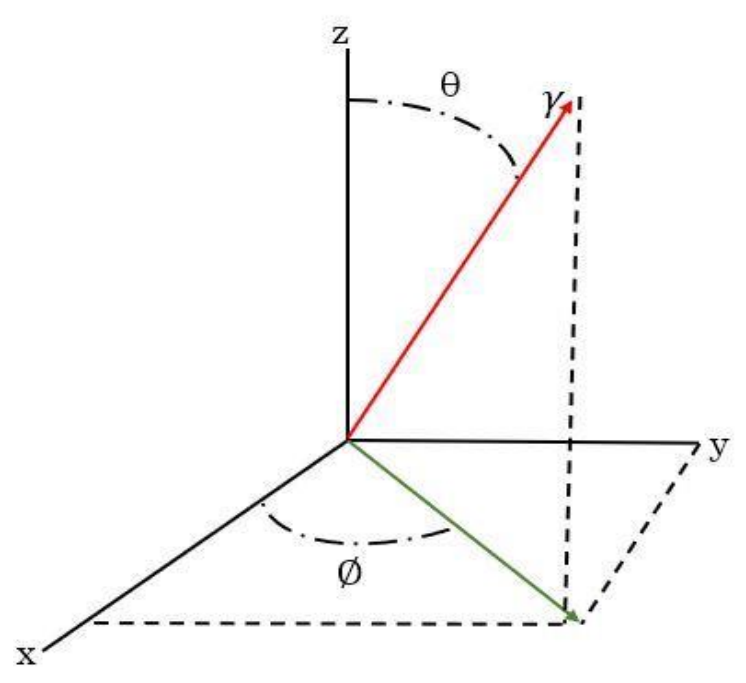

a)

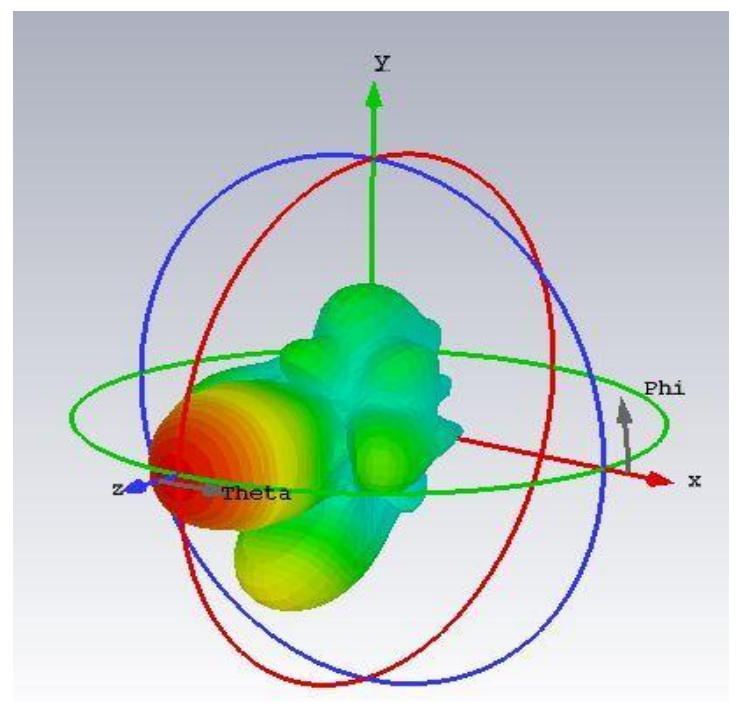

b)

Figura 12. Diagramas de radiación de una antena, a) representación bidimensional y b) representación en un modelo 3D. Fuente: elaboración propia.

\subsection{Eficiencia de radiación}

La eficiencia de radiación es un parámetro para evaluar el rendimiento de una antena, ya que describe qué también una antena irradia la potencia aceptada en su alimentación. La eficiencia es la relación entre la potencia radiada y la potencia entregada a la antena.

La eficiencia total de una antena se usa para tomar en cuenta la pérdida en las terminales de entrada y dentro de la estructura de la antena; dichas pérdidas se pueden deber al desacoplamiento entre la línea de transmisión y la antena y a las pérdidas durante la conducción [31]. Esta eficiencia se describe como (5):

$$
\varepsilon=\frac{P_{\text {radiada }}}{P_{\text {entrada }}}
$$

\subsection{Efecto de las ranuras}

Las ranuras en el parche radiante obligan a las corrientes de superficie a serpentear la estructura aumentando artificialmente la longitud eléctrica de la antena [26]. Las ranuras pueden mejorar el rendimiento de la radiación de la antena al suprimir los modos de orden superior y mejorar la operación de banda ancha [32].

Cuando una antena es conectada a elementos no lineales se producen armónicos en la señal, estos armónicos regresan a la antena y producen un efecto de onda reflejada, disminuyendo así la eficiencia de la antena. Para evitar este inconveniente se utilizan filtros pasa-banda, que eliminan los armónicos no deseados. De igual modo, las ranuras son otra alternativa para eliminar armónicos no deseados sin la necesidad de utilizar filtros [33]. Las ranuras se ajustan de tal manera que la frecuencia o el modo fundamental de resonancia incremente o disminuya al igual que los modos de resonancia de orden superior. Las ranuras ayudan a desplazar las frecuencias de resonancia, lo cual se logra ajustando el ancho y largo de cada ranura hasta llegar a un ajuste donde no se perturbe el modo de operación deseado, esto elimina el problema de la onda reflejada y disminuye la perdida de eficiencia. 
En la Tabla 3 se presenta la comparación de antenas simuladas con diferentes substratos junto a la antena propuesta.

Tabla 3. Comparación de antenas simuladas con diferentes substratos vs antena propuesta con AD450 Fuente: elaboración propia.

\begin{tabular}{lcccc}
\hline Parámetros & Antena 1 [34] & Antena 2 [35] & Antena 3 [36] & Antena propuesta \\
\hline Substrato & FR4 & RT Duroid 5870 & Rogers TMM-3 & AD450 \\
Permitividad relativa & 4.60 & 2.33 & 3.27 & 4.50 \\
Tangente de pérdidas & 0.02 & 0.0012 & 0.002 & 0.0035 \\
Espesor del substrato (mm) & 0.800 & 0.254 & 0.381 & 3.00 \\
Frecuencia (GHz) & 5.3 & 5.7 & 5.7 & $1.86-4.16$ \\
Dimensión (mm) & $28 \times 22$ & - & $42.4 \times 42.4$ & $90 \times 81.4$ \\
Estructura & Planar & Planar & Planar & Planar \\
Ganancia (dB) & 3.1 & 10.28 & 5.85 & $2.48-7.66$ \\
\hline
\end{tabular}

Existen diferentes tecnologías para recolectar energía del medio ambiente, como energía solar, energía eólica, energía térmica, energía cinética, etc. Entre estos esta la energía electromagnética, que abunda en el medio ambiente y se puede recolectar sin limitaciones debido a que las ondas electromagnéticas provienen de una variedad de fuentes, como estaciones de satélite, internet inalámbrico, estaciones de radio y transmisiones digitales. Un sistema de recolección de energía de radiofrecuencia puede capturar y convertir energía electromagnética en un voltaje de corriente continua utilizable [13]. Esto se puede lograr utilizando antenas de microcinta debido a su bajo costo y fácil fabricación.

\section{CONCLUSIONES}

En este documento se realiza la simulación de una antena de alta directividad y de buena ganancia, con un patrón de radiación que permanece en forma directiva, el espesor del sustrato ejerce una separación considerable entre el parche radiante y el plano de tierra, esto genera la disminución de pérdidas en el patrón de radiación. La influencia de las ranuras, a su vez, genera varias frecuencias de resonancia que no se notaban en el diseño original.

Las antenas de microcinta son ideales para la recolección de energía $R F$, sus propiedades y características de diseño permiten obtener antenas de buena ganancia y directividad para los casos donde se tenga una línea de vista directa de la fuente emisora. Cuando se desconoce la fuente emisora son ideales antenas poco directivas, con buen ancho de banda y omnidireccionales para recolectar energía de varias fuentes cercanas.

Las ranuras modifican la distribución de corriente de la antena y aumentan la longitud eléctrica de esta. Aunque los cambios en la posición de la inserción de alimentación alteran las impedancias de entrada en las frecuencias duales, estas frecuencias permanecerán casi constantes. La perturbación en las distribuciones de corriente de superficie aumenta con la longitud de las ranuras.

La antena diseñada permite la realización de la combinación espacial de señales lo cual hace posible prescindir de un combinador de potencia, permitiendo así reducir las pérdidas de potencia que tienen lugar en los transmisores. 


\section{AGRADECIMIENTOS}

Este documento se deriva de un trabajo de tesis de maestría, por lo tanto, no contó con apoyo financiero.

\section{CONFLICTOS DE INTERÉS DE LOS AUTORES}

Los autores declaramos no tener conflictos de interés.

\section{CONTRIBUCIÓN DE LOS AUTORES}

Edison Andrés-Zapata Ochoa: contribuyó a la redacción, análisis y conceptualización. Francisco López-Giraldo: contribuyó a la conceptualización, revisión y supervisión.

Germán David-Goéz: contribuyó a la conceptualización, revisión y supervisión.

\section{REFERENCIAS}

[1] H. F. Bermúdez-Orozco; E. Astaiza-Hoyos; L. F. Muñoz-Sanabria, "Cambios del patrón de radiación en arreglos lineales de dipolos de microcinta a $2,4 \mathrm{GHz}$ en presencia de elementos parásitos," TecnoLógicas, vol. 18, no. 35, pp. 21-34, Aug. 2015. https://doi.org/10.22430/22565337.185

[2] J. Chen; K. Fai Tong; A. Al-Armaghany; J. Wang, "A Dual-Band Dual-Polarization Slot Patch Antenna for GPS and Wi-Fi Applications," IEEE Antennas Wirel. Propag. Lett., vol. 15, pp. 406-409, Jun. 2015. https://doi.org/10.1109/lawp.2015.2448536

[3] H. Elsadek; D. M. Nashaat, "Multiband and UWB V-shaped antenna configuration for wireless communications applications," IEEE Antennas Wirel. Propag. Lett., vol. 7, pp. 89-91, May. 2008. https://doi.org/10.1109/lawp.2007.900953

[4] S. Liu; W. Wu; D. Gang Fang, "Single-Feed Dual-Layer Dual-Band E-Shaped and U-Slot Patch Antenna for Wireless Communication Application,” IEEE Antennas Wirel. Propag. Lett., vol. 15, no. 2, pp. 468-471, Jul. 2015. https://doi.org/10.1109/lawp.2015.2453329

[5] M. Rostamzadeh; S. Mohamadi; J. Nourinia; Ch. Ghobadi; M. Ojaroudi, "Square monopole antenna for UWB applications with novel rod-shaped parasitic structures and novel V-shaped slots in the ground plane," IEEE Antennas Wirel. Propag. Lett., vol. 11, pp. 446-449, Apr. 2012. https://doi.org/10.1109/lawp.2012.2193866

[6] N. M. Awad; M. K. Abdelazeez, "Multislot microstrip antenna for ultra-wide band applications," J. King Saud Univ. Sci., vol. 30, no. 1, pp. 38-45, Jan. 2018. https://doi.org/10.1016/j.jksues.2015.12.003

[7] S. Weigand; G. H. Huff; K. H. Pan; J. T. Bernhard, "Analysis and design of broad-band single-layer rectangular U-slot microstrip patch antennas," IEEE Trans. Antennas Propag., vol. 51, no. 3, pp. 457-468, May. 2003. https://doi.org/10.1109/tap.2003.809836

[8] M. Joler; J. Kucan, "Impact of Slot Parameters on the Three Resonant Frequencies of a Rectangular Microstrip Antenna: Study of the impact of the slot length, width, and position," IEEE Antennas Propag. Mag., vol. 57, no. 4, pp. 48-63, Aug. 2015. https://doi.org/10.1109/map.2015.2453888

[9] S. Chhawchharia; S. Kumar Sahoo; M. Balamurugan; S. Sukchai; F. Yanine, "Investigation of wireless power transfer applications with a focus on renewable energy," Renew. Sustain. Energy Rev., vol. 91, pp. 888-902, Aug. 2018. https://doi.org/10.1016/j.rser.2018.04.101

[10] T. Benyetho; J. Zbitou; L. El Abdellaoui; H. Bennis; A. Tribak, "A New Fractal Multiband Antenna for Wireless Power Transmission Applications," Act. Passiv. Electron. Components, vol. 2018, pp. 1-10, Mar. 2018. https://doi.org/10.1155/2018/2084747

[11] H. Joon Kim; H. Hirayama; S. Kim; K. Jin Han; R. Zhang; J. Woong Choi, "Review of Near-Field Wireless Power and Communication for Biomedical Applications,” IEEE Access, vol. 5, pp. 21264-21285, Sep. 2017. https://doi.org/10.1109/access.2017.2757267

[12] R. Hussein; H. A. Atallah; S. Hekal; A. B. Abdel-Rahman, "A new design for compact size wireless power transfer applications using spiral defected ground structures," Radioengineering, vol. 27, no. 4, pp. 1032- 
1037, 2018. https://doi.org/10.13164/re.2018.1032

[13] L. G. Tran; H. K. Cha;W. T. Park, "RF power harvesting: a review on designing methodologies and applications," Micro Nano Syst. Lett., vol. 5, Feb. 2017. https://doi.org/10.1186/s40486-017-0051-0

[14] H. S. Deshpande; K. J. Karande, "A planar microstrip RF energy harvester 3D cube antenna for multiple frequencies reception," in Conference on Advances in Signal Processing, CASP 2016, Pune, 2016, pp. 327331. https://doi.org/10.1109/casp.2016.7746189

[15] T. A. Elwi, "Novel UWB printed metamaterial microstrip antenna based organic substrates for RF-energy harvesting applications," AEU - Int. J. Electron. Commun., vol. 101, pp. 44-53, Mar. 2019. https://doi.org/10.1016/j.aeue.2019.01.026

[16] N. Shariati; W. S. T. Rowe; K. Ghorbani, "Highly sensitive rectifier for efficient RF energy harvesting," 2014 44th European Microwave Conference, Rome, 2014, pp. 1190-1193. https://doi.org/10.1109/eumc.2014.6986654

[17] J. M. Barcak; H. P. Partal, "Efficient RF energy harvesting by using multiband microstrip antenna arrays with multistage rectifiers," in 2012 IEEE Subthreshold Microelectronics Conference, SubVT, Waltham, 2012. https://doi.org/10.1109/subvt.2012.6404327

[18] Y. Zhou; C. Huerta; J. Hinojosa, "Three-band ambient wireless energy harvesting system,"2016 IEEE International Symposium on Antennas and Propagation (APSURSI), Fajardo, 2016, pp. 613614. https://doi.org/10.1109/aps.2016.7696015

[19] Z. Popovic et al., "Scalable RF energy harvesting," IEEE Trans. Microw. Theory Tech., vol. 62, no. 4, pp. 1046-1056, Feb. 2014. https://doi.org/10.1109/tmtt.2014.2300840

[20] L. L. Pon; S. K. Abdul Rahim; C. Yen Leow; M. Himdi; M. Khalily, "Displacement-tolerant printed spiral resonator with capacitive compensated-plates for non-radiative wireless energy transfer," IEEE Access, vol. 7, pp. 10037-10044, Jan. 2019. https://doi.org/10.1109/access.2019.2891015

[21] A. Rajagopalan; A. K. Ramrakhyani; D. Schurig; G. Lazzi, "Improving power transfer efficiency of a shortrange telemetry system using compact metamaterials," IEEE Trans. Microw. Theory Tech., vol. 62, no. 4, pp. 947-955, Feb. 2014. https://doi.org/10.1109/tmtt.2014.2304927

[22] J. Zhang; X. Yuan; C. Wang; Y. He, "Comparative Analysis of Two-Coil and Three-Coil Structures for Wireless Power Transfer," IEEE Trans. Power Electron., vol. 32, no. 1, pp. 341-352, Feb. 2016. https://doi.org/10.1109/tpel.2016.2526780

[23] M. Fantuzzi; D. Masotti; A. Costanzo, "A Novel Integrated UWB-UHF One-Port Antenna for Localization and Energy Harvesting," IEEE Trans. Antennas Propag., vol. 63, no. 9, pp. 3839-3848, Jul. 2015. https://doi.org/10.1109/tap.2015.2452969

[24] E. Gómez Rodríguez; I. Rodríguez Prieto; F. Marante Rizo; L. Rizo Salas, "Estudio de la variación de diferentes parámetros en antenas de microcinta AAPC," Ing. Electrónica, Automática y Comun., vol. 34, no. 1, pp. 27-39, Jan. 2013. http://scielo.sld.cu/scielo.php?script=sci_arttext\&pid=S1815-59282013000100003

[25] M. Kumar Khandelwal; B. Kumar Kanaujia; S. Kumar, "Defected ground structure: Fundamentals, analysis, and applications in modern wireless trends," International Journal of Antennas and Propagation, vol. 2017, Feb. 2017. https://doi.org/10.1155/2017/2018527

[26] N. Singh; B. K. Kanaujia; M. T. Beg; Mainuddin; T. Khan; S. Kumar, "A dual polarized multiband rectenna for RF energy harvesting," AEU - Int. J. Electron. Commun., vol. 93, pp. 123-131, Sep. 2018. https://doi.org/10.1016/j.aeue.2018.06.020

[27] A. Benayad; M. Tellache, "A compact energy harvesting multiband rectenna based on metamaterial complementary split ring resonator antenna and modified hybrid junction ring rectifier," Int. J. RF Microw. Comput. Eng., vol. 30, no. 2, pp. 1-11, Feb. 2020. https://doi.org/10.1002/mmce.22031

[28] A. E. Hidalgo; F. M. Rizo, "Microstrip antenna with metamaterial hybrid structure for 2.4 GHz," Ingeniare, vol. 27, no. 1, pp. 22-33, Mar. 2019. https://doi.org/10.4067/s0718-33052019000100022

[29] A. A. Deshmukh; K. P. Ray, "Formulation of resonance frequencies for dual-band slotted rectangular microstrip antennas," IEEE Antennas Propag. Mag., vol. 54, no. 4, pp. 78-97, Sep. 2012. https://doi.org/10.1109/map.2012.6309159

[30] J. Balcells; Y. Damgaci; B. A. Cetiner; J. Romeu; L. Jofre, "Polarization reconfigurable MEMS-CPW antenna for mm-wave applications," EuCAP 2010 - 4th Eur. Conf. Antennas Propag., Barcelona, 2010. pp. 1856. https://ieeexplore.ieee.org/abstract/document/5505012

[31] F. Sarrazin; S. Pflaum; C. Delaveaud, "Radiation Efficiency Improvement of a Balanced Miniature IFAInspired Circular Antenna," IEEE Antennas Wirel. Propag. Lett., vol. 16, pp. 1309-1312, Nov. 2016. https://doi.org/10.1109/lawp.2016.2633308

[32] B. Rao Perli; A. Maheswara Rao, "Analysis of microstrip patch antenna with loading slot using characteristic modes," 2020 7th Int. Conf. Smart Struct. Syst. ICSSS, Chennai, 2020, pp. 2-5. https://doi.org/10.1109/icsss49621.2020.9202029

[33] S. Á. Jaramillo-Flórez, "Filtros a Frecuencias de Microondas con Doble Resonador en Anillo Elípticos Confocales," TecnoLógicas, p. 517- 528, Nov. 2013. https://doi.org/10.22430/22565337.345 
[34] M. Moubadir; I. Badaoui; N. A. Touhami; M. Aghoutane; M. El Ouahabi, "A new circular polarization dual feed microstrip square patch antenna using branch coupler feeds for WLAN/HIPERLAN applications," Procedia Manufacturing, vol. 32, pp. 702-709, 2019. https://doi.org/10.1016/j.promfg.2019.02.274

[35] C. Wnng; K.Chang, "A novel CP patch antenna with a simple feed structure," IEEE Antennas and Propagation Society International Symposium. Transmitting Waves of Progress to the Next Millennium. 2000 Digest. Held in conjunction with: USNC/URSI National Radio Science Meeting (C, Salt Lake City, 2002, pp. 1000-1003. https://doi.org/10.1109/aps.2000.875389

[36] T. Kingsuwannaphong; V. Sittakul, "Compact circularly polarized inset-fed circular microstrip antenna for $5 \mathrm{GHz}$ band," Comput. Electr. Eng., vol. 65, pp. 554-563, Jan. 2018. https://doi.org/10.1016/j.compeleceng.2017.02.027 